\title{
Article \\ Comparison of Small Unmanned Aerial Vehicles Performance Using Image Processing
}

\author{
Esteban Cano, Ryan Horton, Chase Liljegren and Duke M. Bulanon * \\ Department of Physics and Engineering, Northwest Nazarene University, Nampa, ID 83686, USA; \\ ecano@nnu.edu (E.C.); ryanhorton@nnu.edu (R.H.); cliljegren@nnu.edu (C.L.) \\ * Correspondence: dbulanon@nnu.edu; Tel.: +1-208-467-8047
}

\begin{abstract}
Precision agriculture is a farm management technology that involves sensing and then responding to the observed variability in the field. Remote sensing is one of the tools of precision agriculture. The emergence of small unmanned aerial vehicles (sUAV) have paved the way to accessible remote sensing tools for farmers. This paper describes the comparison of two popular off-the-shelf sUAVs: 3DR Iris and DJI Phantom 2. Both units are equipped with a camera gimbal attached with a GoPro camera. The comparison of the two sUAV involves a hovering test and a rectilinear motion test. In the hovering test, the SUAV was allowed to hover over a known object and images were taken every second for two minutes. The position of the object in the images was measured and this was used to assess the stability of the sUAV while hovering. In the rectilinear test, the sUAV was allowed to follow a straight path and images of a lined track were acquired. The lines on the images were then measured on how accurate the sUAV followed the path. Results showed that both SUAV performed well in both the hovering test and the rectilinear motion test. This demonstrates that both sUAVs can be used for agricultural monitoring.
\end{abstract}

Keywords: agriculture; digital image processing; machine vision; precision agriculture; unmanned aerial vehicle (UAV)

\section{Introduction}

For a farmer, knowing the health and current state of the year's crop is essential for an effective crop management. One of the tools that a farmer can use to effectively manage his crops is precision agriculture. Precision agriculture is a technique in which a farmer uses observation and measurement with the aid of newly available technologies to know how to manage their crops in the most proficient way possible [1, 2]. With the acquired information, a farmer can do site specific crop management in which a particular section of a field can be given individualized treatment for their specific health. Observations and measurements of a crop can be taken in many ways, but in all cases, the findings would lead to the knowledge of how to best treat and manage the plants for maximum yield. Precision agriculture is useful for farmers due to its very straight forward and correlative outcomes achieved from simply observing, measuring, and analyzing the field and then managing it accordingly. Technologies such as yield monitoring and variable rate applications are commonly used precision agriculture tools [3]. Precision agriculture can not only produce better crop productivity by tending to each plant's individual conditions, but also provides means to more efficient distribution of resources (e.g. water, fertilizer, and pesticides), leading to more profitable and ecofriendly farms.

Although precision agriculture encompasses many different methods of data acquisition, today's technology allows for remote sensing techniques to act as an extremely useful tool for farmers. Remote sensing is an approach in which information is obtained without requiring a person to be physically present to collect the data. Remote sensing combined with Geographic Information Systems (GIS) and Global Positioning Systems (GPS) provide farmers the technologies needed to maximize the output of their crops $[4,5]$. Therefore, with use of technology data can be remotely collected and composed to be analyzed by means of unmanned vehicles, satellites, and sensors. This 
manner of observation is a huge innovation for the agriculture industry by allowing farmers access to forms of observation and measurements, as well as an abundance of information that could not have been had prior to remote sensing. With the use of remote sensing, each plant's state of health amongst an entire crop can more easily be recognized from large amounts of data that can be collected and stored and later analyzed. In addition, remote sensing technologies has the added benefit of reducing environmental impacts [6].

There are a number of ways to implement remote sensing. These include satellite imaging, imaging using manned or unmanned aerial vehicles (UAV), and imaging using ground-based systems [7]. In a day when small unmanned aerial vehicles (sUAV) are becoming much more common and influential in numerous industrial fields [8], it is no surprise that they can be utilized in agriculture applications as well $[9,10]$. A comparative study between using sUAV and manned aircraft to image citrus tree conditions showed that sUAV produced higher spatial resolution images [11]. One of the most useful yet affordable remote sensing systems can now be obtained with the purchase of a small quadcopter or drone which can then be flown over a farmer's desired agriculture fields. The drones can then take images of the farmer's crop with a variety of camera filters to provide the farmer with multiple spectrums of imaging. Not only is a sUAV useful in providing current aerial images of their entire crop, but it also allows for the opportunity for image processing and analysis which can give even more information of the health their crops as well as identifying areas of the crop that require specific forms of attention. A study by Bulanon et al. [12] to evaluate different irrigation systems of an apple orchard used combination of sUAV and image processing. The small drones can be easily flown and maintained with little training making them a great option for farmer's looking to further their farming by merging agriculture with the technology of remote sensing.

With the price of remote sensing sUAVs becoming much more affordable and thus a realistic application for today's farmers, the research done in this paper strives to test, analyze, and compare two of today's popular off-the-shelf ready-to-fly sUAVs in multiple aerial competence tests and evaluate their suitability for agricultural use. The objectives of this paper are:

1. To compare the flight performance of two off-the-shelf sUAVs: 3DR Iris and DJI Phantom 2,

2. To develop image processing algorithms to evaluate the performance of the two sUAVs

3. To demonstrate an agricultural application of the sUAV.

\section{Materials and Methods}

\subsection{Small Unmanned Aerial Vehicles}

Unmanned aerial vehicles (UAV) can be classified according to size. The classification includes micro UAV, small UAV, medium UAV, and large UAV [13]. The micro UAVs are extremely small in size and applies to sizes of about an insect to $30-50 \mathrm{~cm}$ long. The small UAV (sUAV) are UAVs with dimension greater than $50 \mathrm{~cm}$ and less than $2 \mathrm{~m}$. The Federal Aviation Agency defines sUAV as an aircraft that weighs more than $0.25 \mathrm{~kg}$ but less than $25 \mathrm{~kg}$ [14]. The medium UAVs have dimension ranging from $5 \mathrm{~m}$ to $10 \mathrm{~m}$ and can carry payloads of up to $200 \mathrm{~kg}$, while large UAVs applies to the UAVs used mainly for combat operations by the military. In this paper the focus is on sUAV and its application to agriculture. While most people are able to build their own sUAV using do-it-yourself kits, off-the-shelf ready-to-fly sUAV are also available. The advantages of the off-the-shelf sUAV are they are ready to fly and there is not much tuning involved as compared with DIY kits. In addition, these off-the-shelf sUAV comes with camera gimbals that could then be easily used for agricultural surveying. Two of the most popular sUAV in the market were used in this study: 1) 3DR Iris [15] and 2) DJI Phantom 2 [16]. Some specifications of the two drones is provided in the Table 1. An image of the 3DR Iris is shown in Figure 1 and is noticeably wider than the DJI Phantom 2 which is pictured in Figure 2. The greater width of the Iris makes it so that the distance between the front and back props is less than the distance from side to side. The DJI Phantom 2 in contrast provides prop 
locations that are symmetrically set in a square around the center of the drone. Both of the drones used functionally similar gimbals to operate a Go-Pro camera for in flight imaging.

Table 1: Iris and Phantom 2 Specifications

\begin{tabular}{ccc}
\hline & IRIS & Phantom 2 \\
\hline Motors & 4 & 4 \\
Max Payload & $400 \mathrm{~g}$ & $300 \mathrm{~g}$ \\
Flight Time & $16-22 \mathrm{~min}$ & $14-25 \mathrm{~min}$ \\
Max Flight Speed & $22.7 \mathrm{~m} / \mathrm{s}$ & $15 \mathrm{~m} / \mathrm{s}$ \\
Motor to Motor Dimensions & $550 \mathrm{~mm}$ & $350 \mathrm{~mm}$ \\
Flight Controller & Pixhawk & NASA-MV2 \\
Software (Ground Station) & Mission Planner & DJI Ground Station \\
Flight Modes & Manual & Manual \\
& Hover & Hover \\
& Auto & Auto \\
Battery & $5100 \mathrm{mAh}$ & $5200 \mathrm{mAh}$ \\
Gimbal & Tarot Go-Pro Gimbal & DJI Go-Pro Gimbal \\
\hline
\end{tabular}

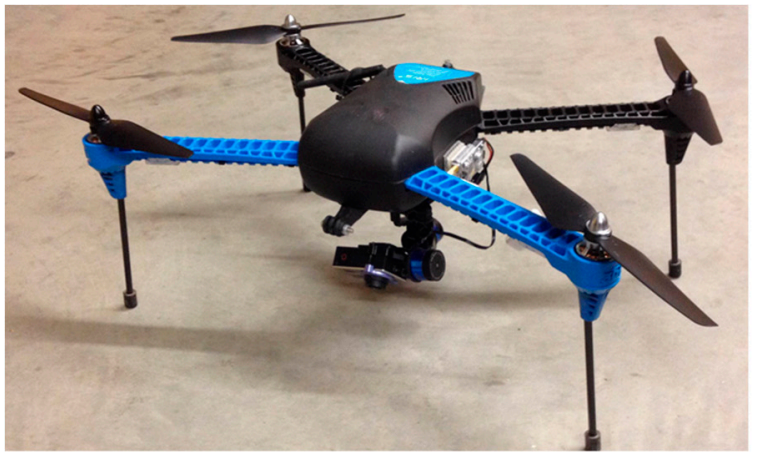

a) 3DR I ris

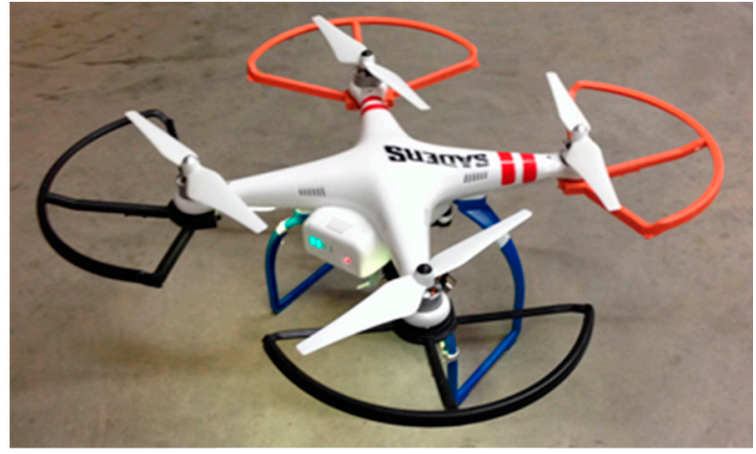

b) DJI Phantom 2

Figure 1. 3DR Iris and DJI Phantom 2 quadcopter drones.

\subsection{Image Acquisition for Agricultural Application}

The image acquisition system used for both sUAV was a modified GoPro Hero 3 camera. The GoPro Hero 3 can shoot both videos and pictures. In this paper, the camera was used to acquire images. The camera was set to capture 11 megapixels resolution and the white balance was set to $5500 \mathrm{~K}$. The camera was modified from an RGB camera to a near-infrared, green, and blue (NGB) camera and this enabled the capture of near-infrared, green, and blue bands in the same image. The camera modification steps included the removal of the infrared-blocking filter of the GoPro and replacing the filter with a Schott BG3 filter, which blocks the red band but passes near-infrared, green, and blue. Figure 2 shows the modified camera and the resulting images. The modified images shows the plants having a reddish hue as compared with the normal greenish hue that we observe in the RGB image. For the RGB image, the green color of the plants are caused by the leaves' pigments reflecting the green light and absorbing the red and blue lights. For the modified image, the high amount of red reflection from the plants in the modified image is due to the fact that plants with more chlorophyll will reflect more near-infrared energy. The modified camera was used for the comparison tests. 


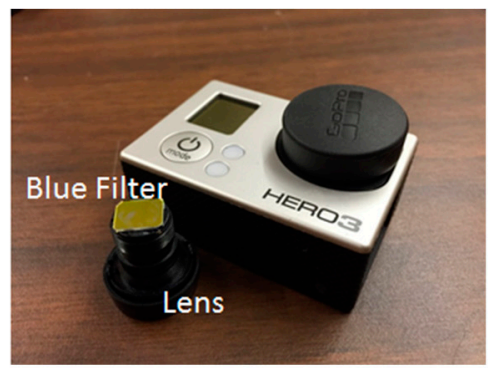

a) Modified GoPro Camera

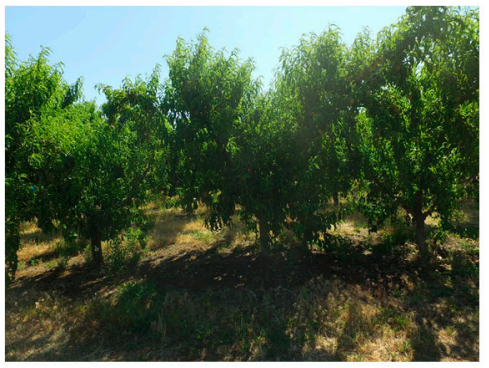

b) Unmodified GoPro Image

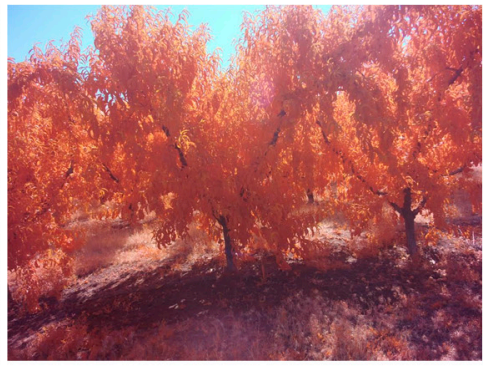

c) Modified GoPro Image

Figure 2. Modified GoPro Camera and resulting images.

\subsection{Performance Evaluation Tests}

The application of sUAV for agricultural surveying involves taking a single picture at a certain altitude or taking multiple pictures following a waypoint path generated by the user. Based on these applications, two performance evaluation tests were conducted to compare the two off-the-shelf sUAVs. These tests were the hovering test and rectilinear motion test. In both of these tests, a GoPro camera is attached to the sUAV camera gimbal and the camera then takes images. Then the images are used to evaluate the flight performance of the sUAV using image processing and analysis. A similar test to evaluate the performance of an autonomous helicopter while hovering was conducted by Xiang and Tian [17]. However, instead of using image processing, they used the onboard sensors.

\subsubsection{Hovering Test}

In the hovering test, the sUAV was flown over a 2-meter square PVC pipe at three different altitudes: $5 \mathrm{~m}, 15 \mathrm{~m}$, and $25 \mathrm{~m}$. At each altitude, the sUAV was allowed to hover for two minutes and images were taken every 0.5 second. The pixel resolution for each altitude are the following: 116 pixels $/ \mathrm{m}(5 \mathrm{~m}), 71$ pixels $/ \mathrm{m}(15 \mathrm{~m})$, and 51 pixels $/ \mathrm{m}(25 \mathrm{~m})$. The two sUAV were tested at the same time with a wind speed of 2 miles per hour (ESE). The time-lapse images of the PVC square were used to measure stability of the sUAV while hovering. Figure 3 shows one of the hovering test for the DJI Phantom.

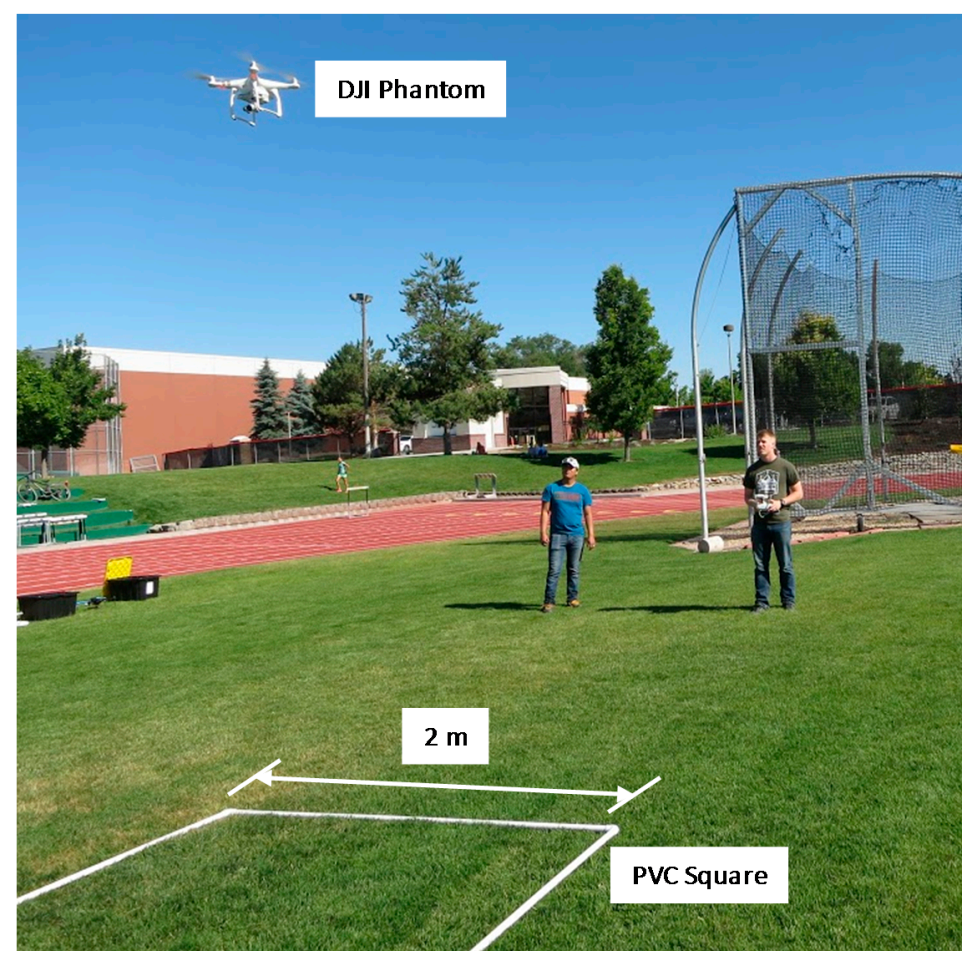

Figure 3. Hovering test for the DJI Phantom. 


\subsubsection{Rectilinear Motion Test}

In the rectilinear motion test, the sUAV was programmed to fly a straight path over the running track at Northwest Nazarene University (NNU). The waypoint path of the sUAV was based on the straight line markings of the running track, which constrains the straight line motion of the sUAV. The wind condition during this test was 4 miles per hour (ESE). Similar to the hovering test, the camera was also programmed to acquire images as it moved over the track every 0.5 second. The acquired images were then used to measure the stability in straight line motion. Figure 4 shows one of the tests for the 3DR Iris.

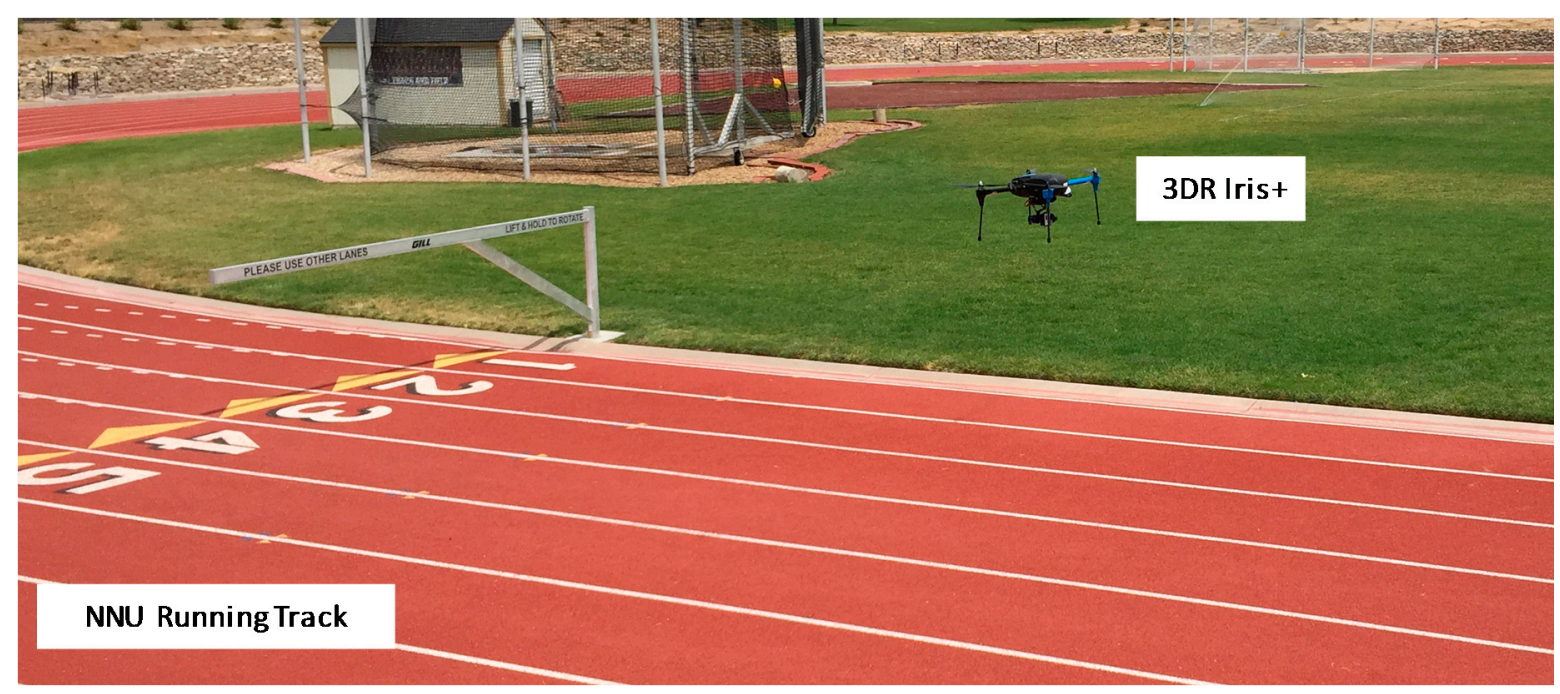

Figure 4. Rectilinear motion test for the 3DR Iris.

\subsection{Image Processing for Performance Evaluation}

\subsubsection{Hovering Test}

To evaluate the hovering test using image processing [18], the center of area of the PVC square inside the image was used as the stability parameter. The stability was measured based on the change of the center of area. Figure 5 demonstrates this concept. The solid black line is the segmented PVC square from the first acquired image and this was used as the set point. The gray lines are the segmented PVC square from the subsequent images. The position of the center of area for each subsequent image were then compared with the set point image, and this was used to evaluate stability in the hovering test.

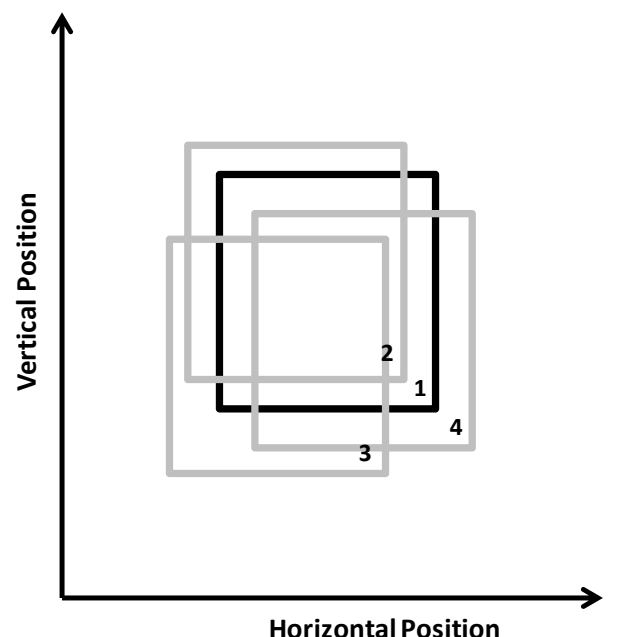

Figure 5. Concept of hovering test evaluation using image processing. 


\subsubsection{Rectilinear Motion Test}

Figure 6 shows the concept of the rectilinear motion test evaluation. The black sUAV is the start position and the square enclosing the sUAV represents the field of view (FOV) of the camera. As the sUAV follows the programmed straight path, the actual position of the sUAV is different from the directed path and these are shown by the gray sUAVs with their respective FOVs. The features in the running track were then used to measure the deviation from the programmed straight path by comparing the line positions from the image acquired from the start position.

\subsection{Monitoring an Experimental Fruit Orchard}

The sUAVs were used to monitor an experimental fruit orchard to demonstrate its surveying capability. The orchard is located at the Parma Research and Extension Center at the University of Idaho. Two different images of the whole orchard were acquired. The first set was a single shot of the whole orchard taken at $91 \mathrm{~m}$ to show the hovering capability. In the second set, a flight plan was created to acquire multiple images and then the images were mosaicked to create a single image of the field. This second set demonstrated the capability of the sUAV to follow the flight path (rectilinear motion).

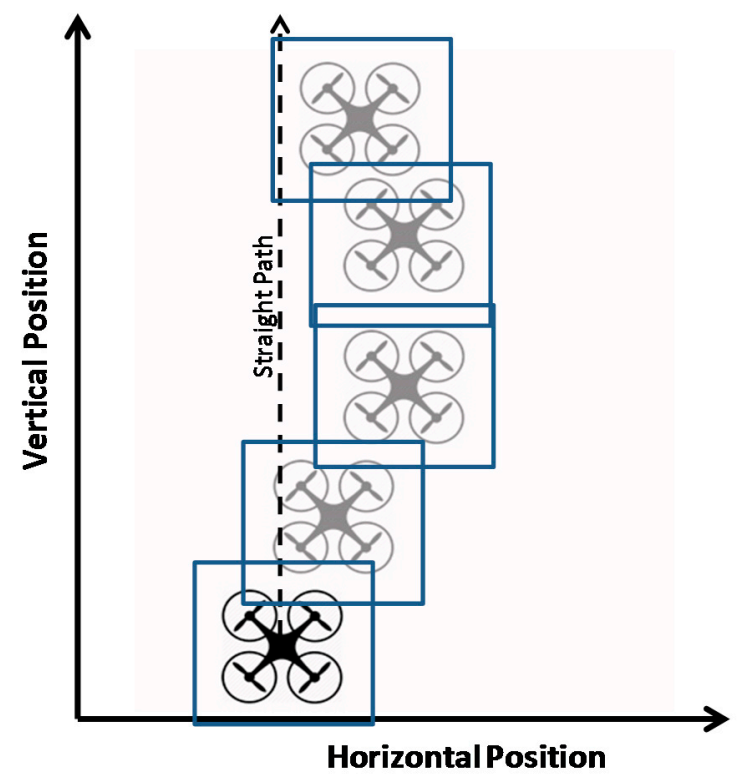

Figure 6. Concept of rectilinear motion test evaluation using image processing.

\section{Results and Discussion}

\subsection{Hovering Test}

Figure 7 shows the original image of the PVC square and the segmented image. The high contrast between the PVC and the grass facilitated the segmentation of the PVC from the background. This image was used as the set point image and the position of the PVC square from the subsequent image was compared to this image. 


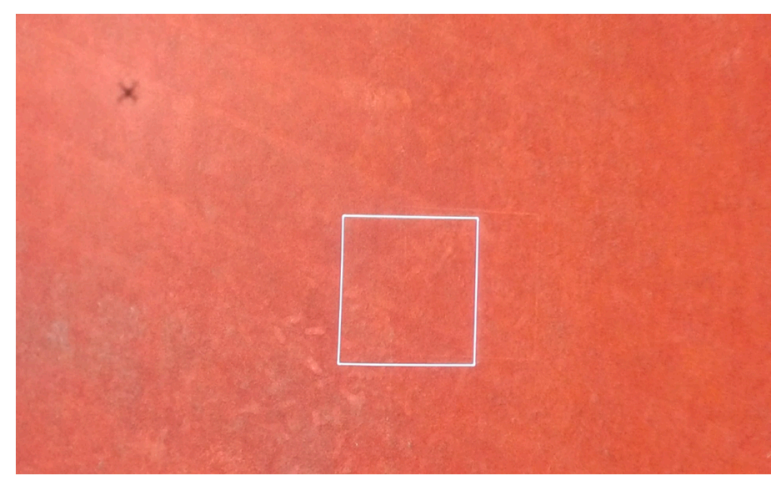

a) Original image

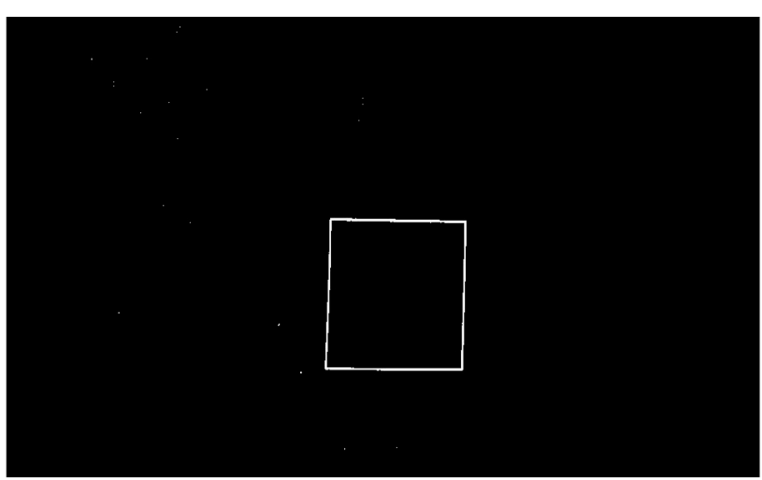

b) Segmented image

Figure 7. Set point image for hovering test.

Figure 8 shows the comparison between the set point image and a subsequent image. The overlaid images show that the position of the PVC square changed, which means that the sUAV is moving even though it is in hover mode. The overlaid images demonstrated that a simple image processing approach could be used to evaluate the stability of the sUAV in hover mode.

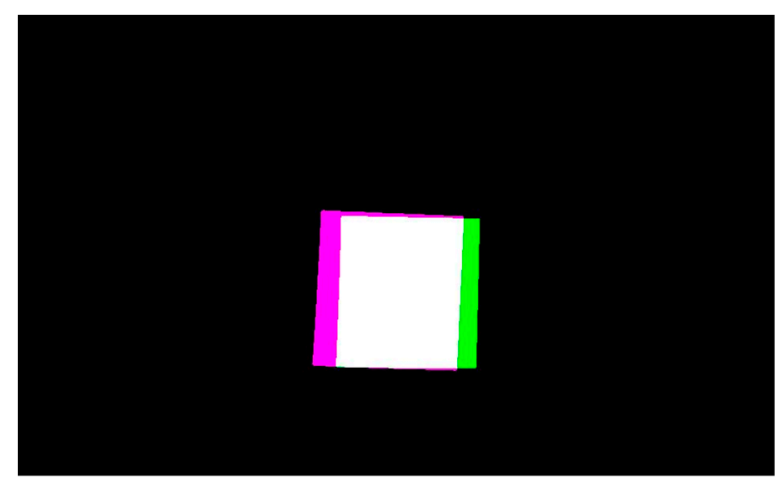

a) Overlay of image 1 and image 2

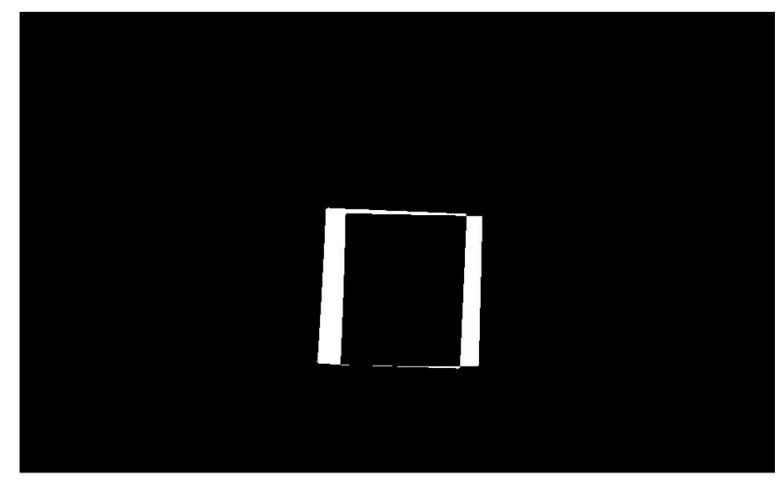

b) Image subtraction of image 1 and 2

Figure 8. Comparison between set point image and subsequent image.

Figure 9 shows the deviation of the 3DR Iris while it is in hover mode at the three different altitudes. This deviation is the difference between the center of area from the set point image and the subsequent images for both the $\mathrm{x}$ and $\mathrm{y}$ axes. The hovering results of the 3DR Iris shows that the maximum deviation is $0.45 \mathrm{~m}$ while hovering at the lowest height of $5 \mathrm{~m}$ and the deviation is $0.4 \mathrm{~m}$ while hovering at $25 \mathrm{~m}$. The deviation decreased as the height is increased because the image resolution of the PVC square decreased. A similar trend can be observed from the hovering results of the DJI Phantom (Figure 10), which showed a maximum deviation of $0.75 \mathrm{~m}$ while hovering at 5 $\mathrm{m}$ and a deviation of $0.35 \mathrm{~m}$ at a hovering height of $25 \mathrm{~m}$. Although, the lower altitude showed the highest deviation, the images had higher spatial resolution. These results will be very useful when performing image mosaicking, which is a process of stitching images to form an image with a much larger field of view. Based from these results, when performing image mosaicking, the image overlaps should take into account the altitude the images are taken at. Larger image overlaps will be accounted for lower heights and smaller overlaps for the higher altitudes. 

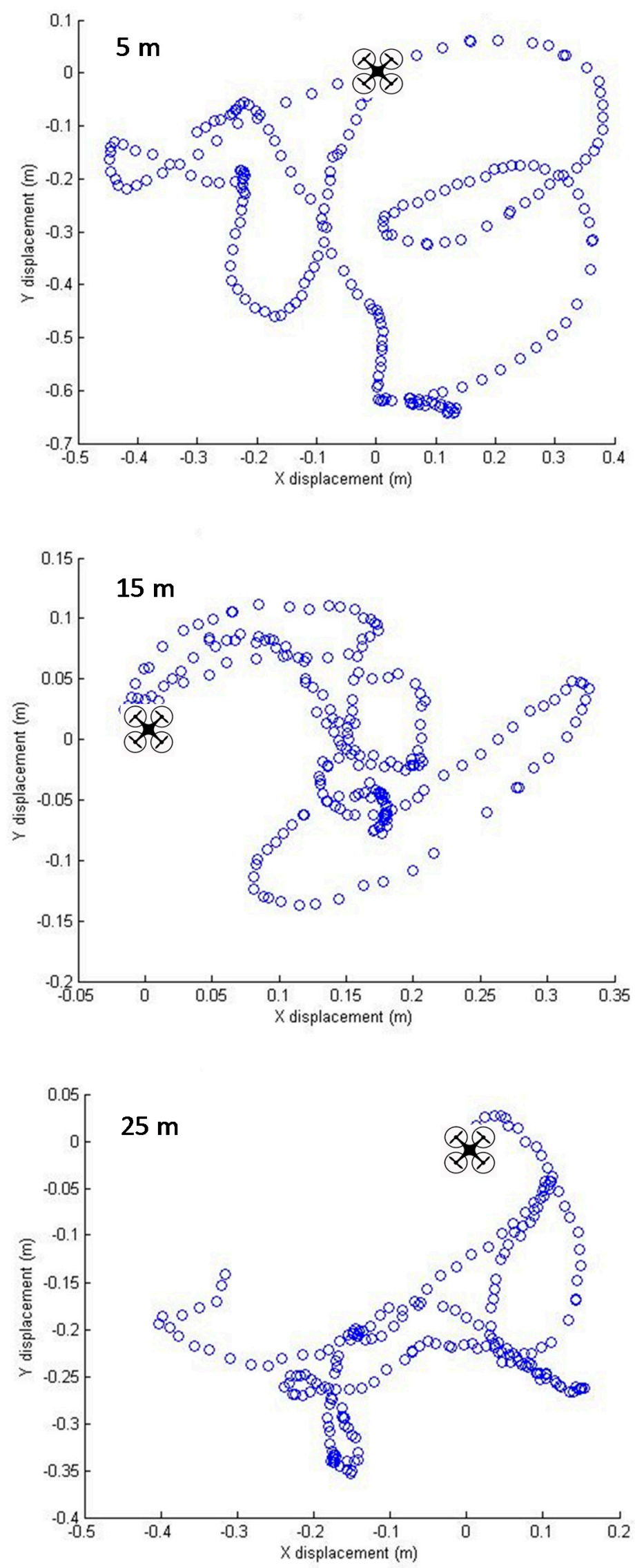

Figure 9. 3DR Iris deviation during hovering test using image processing. 

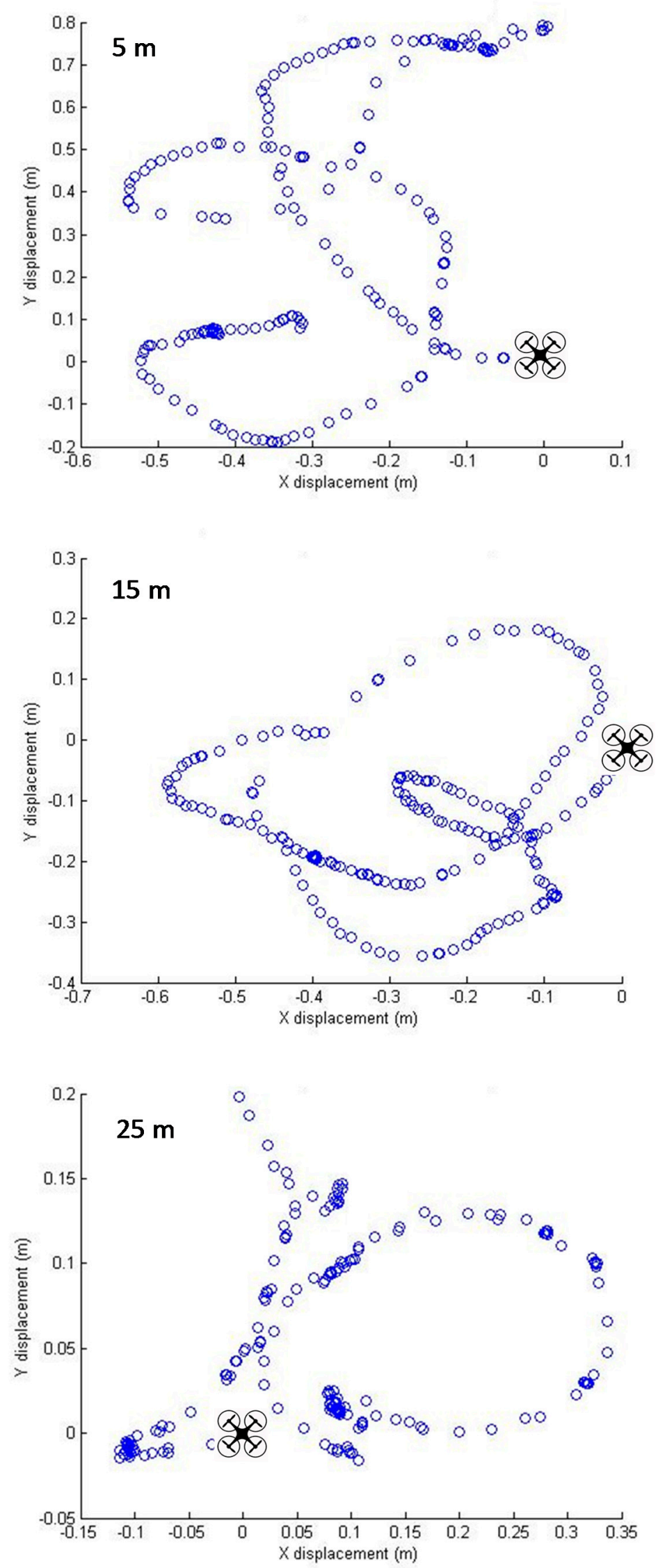

Figure 10. DJI Phantom 2 deviation during hovering test using image processing. 


\subsection{Rectilinear Motion Test}

Figure 11 shows an example of the image processing for evaluating the rectilinear motion test. The first step is to segment the feature that will be used for evaluation. In this case, the feature used was the green bleacher. Thresholding was used to segment the green bleacher because of the high color contrast. After segmentation, a size filter was passed to remove the salt and pepper noise. Following the filtering was an operation to fill the holes and the extraction of the large object in the image which was the bleacher. The position of the bleacher was then used to measure the deviation of the sUAV from the start position.

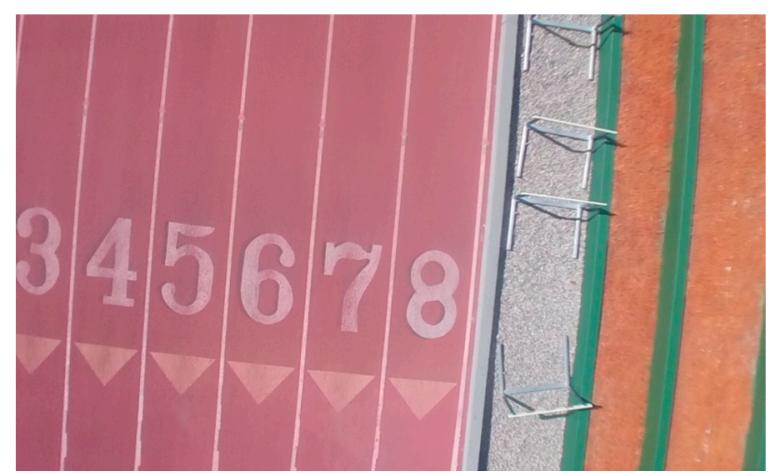

a) Original image

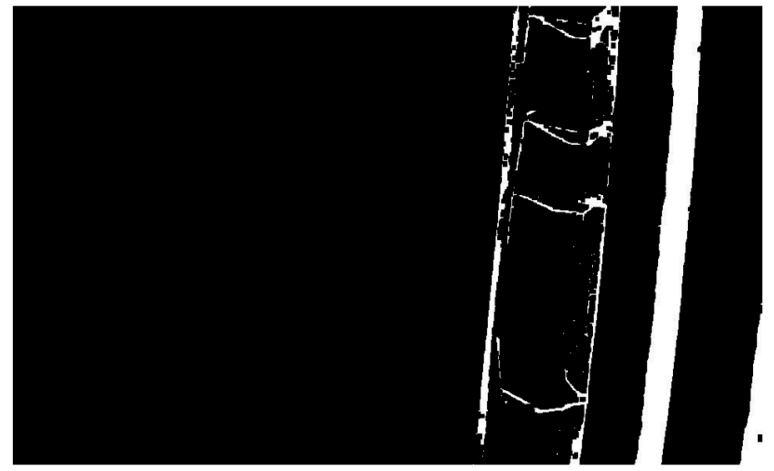

c) Filling holes

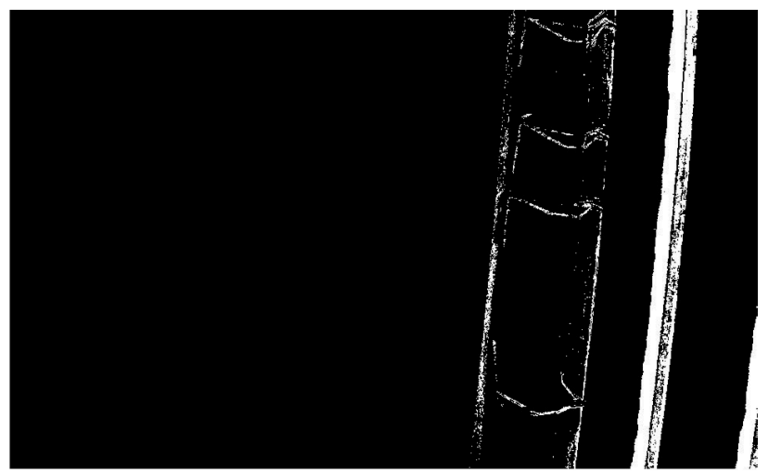

b) Segmented image

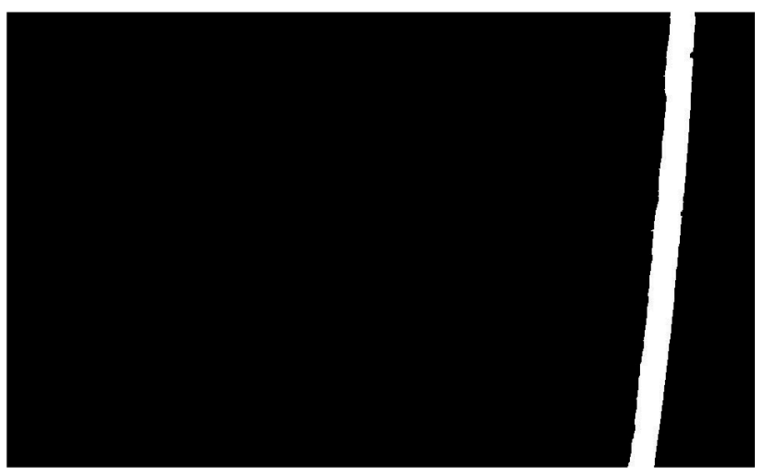

d) Extraction of line

Figure 11. Example image processing for rectilinear motion evaluation.

Figure 12 shows the path of the sUAV calculated using image processing for both sUAV. It can be noted that both sUAVs deviated from the center line and moved with a sinusoidal characteristic, which is typical for a position control system trying to correct itself. For the time that the sUAVs were tested, the maximum deviation from the center line is less than $1 \mathrm{~m}$, which is less than the reported position error for both sUAV. It is also observed that the deviation values for both sUAV are similar to the hover test values. These results show the advancement of the control system of the off-the-shelf sUAV and this demonstrates their capabilities in surveying tasks for agricultural field observation. 

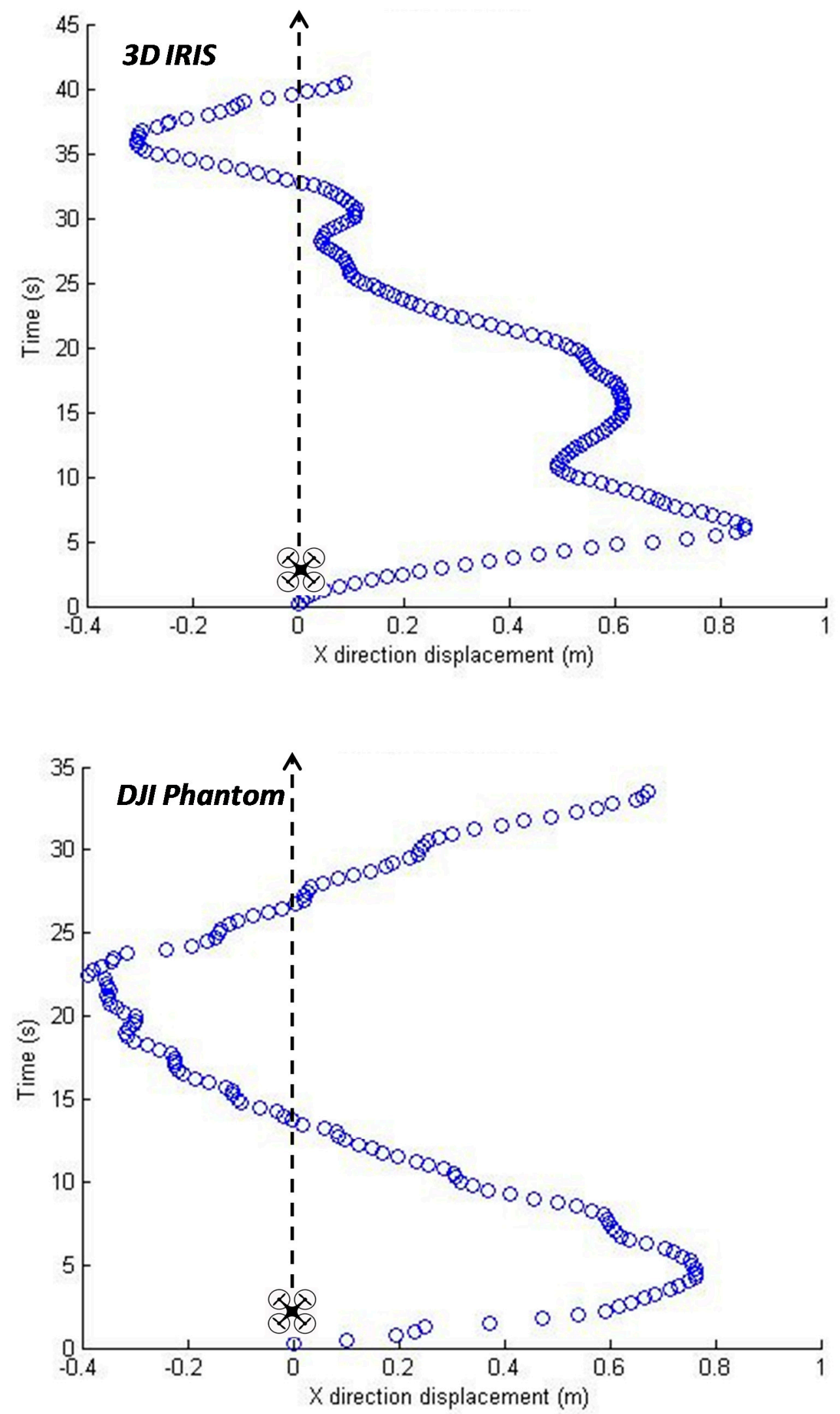

Figure 12. Estimated path of sUAV for rectilinear motion test using image processing.

\subsection{Apple Orchard Monitoring}

Both sUAVs were used to acquire an image of an experimental apple orchard. A single shot of the whole orchard is shown by Figure 13, which was taken by the DJI Phantom. This image was taken 
at an altitude of approximately $91 \mathrm{~m}$. Another set of images were taken to show that the sUAV could be used to follow a flight plan, acquire multiple images with $50 \%$ overlap, and then mosaic the images. Figure 14 shows the mosaicked image. Comparing the single shot and the mosaicked image shows that both sUAV can be used for monitoring agricultural fields.

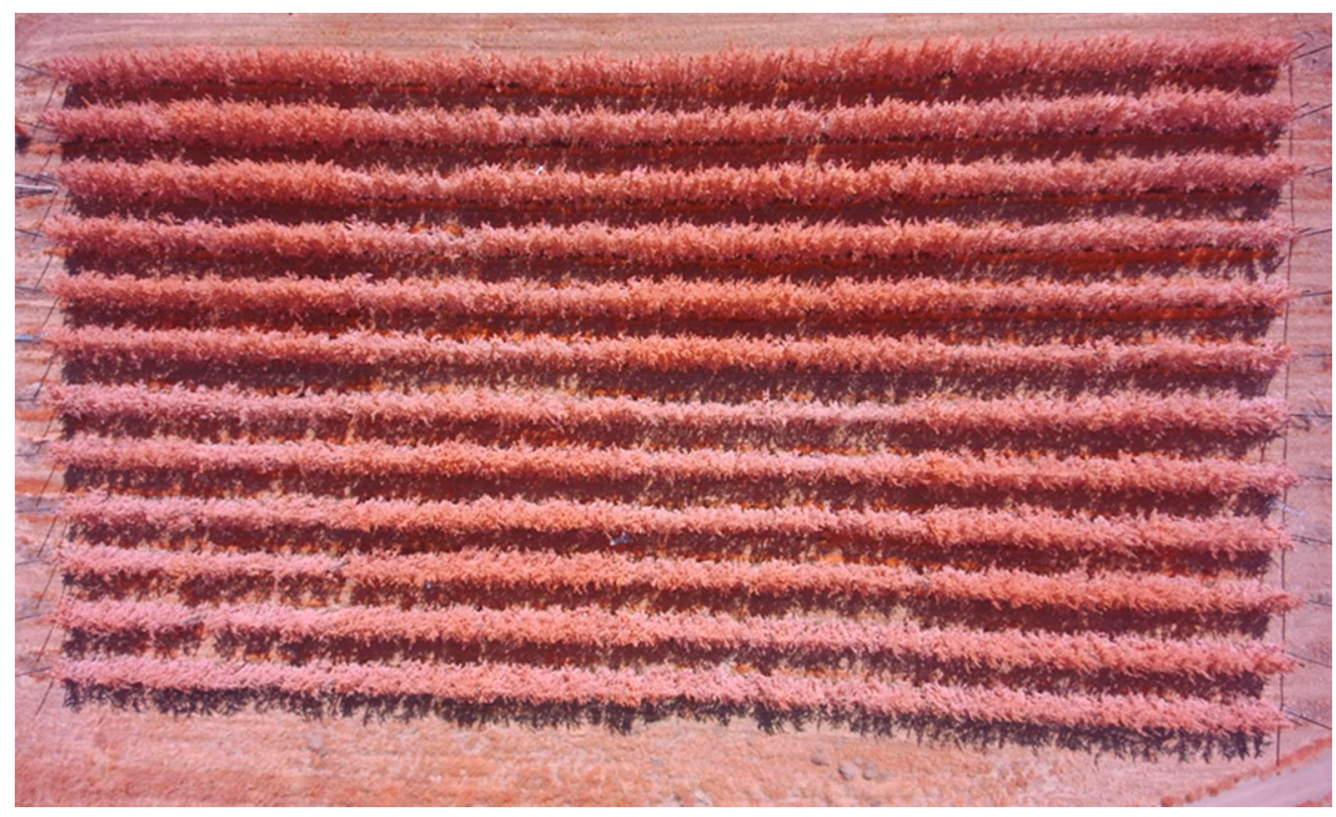

Figure 13. Single shot of apple orchard.

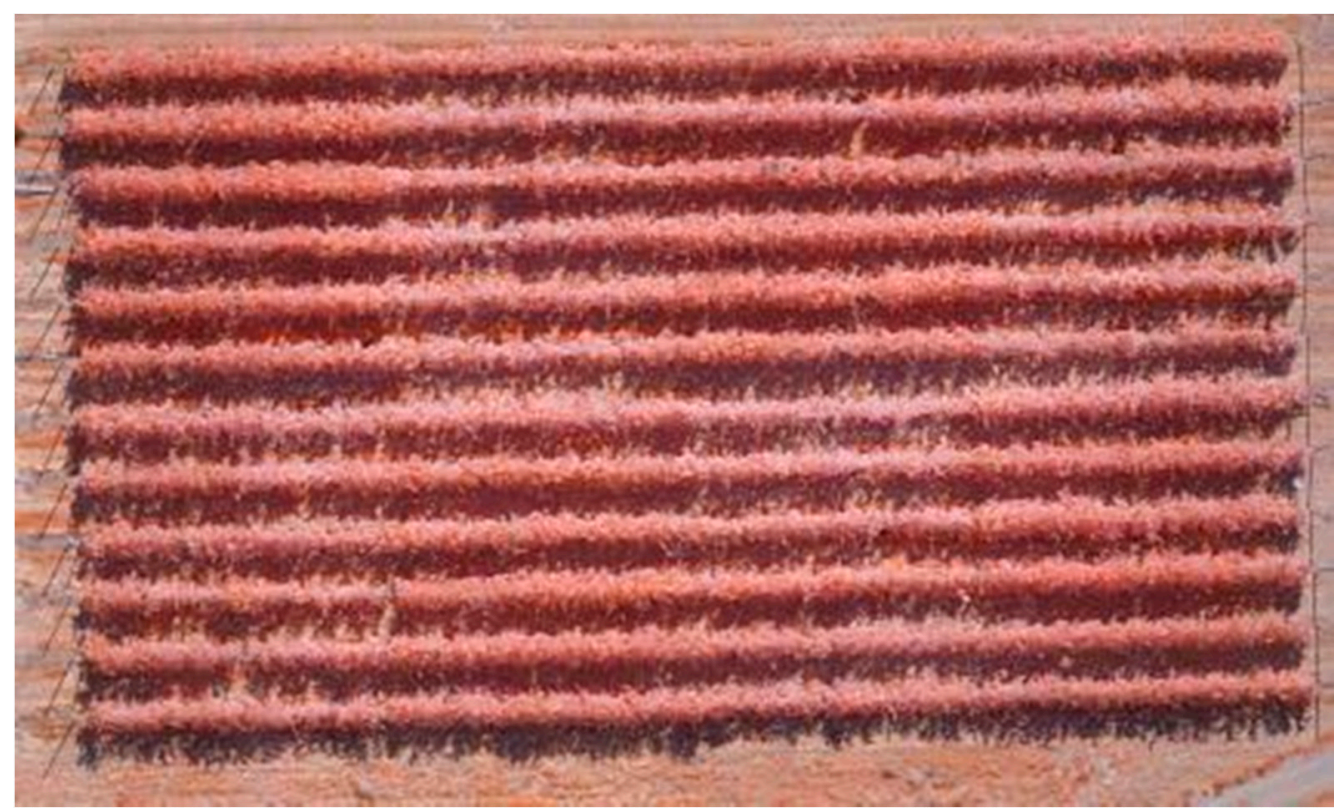

Figure 14. Mosaicked image of apple orchard.

\section{Conclusions}

Two of the popular sUAVs in the market: 3DR Iris and DJI Phantom 2 were compared and evaluated using image processing. The comparison included the hovering test and the rectilinear motion test. For the hovering test, the sUAV took images of an object and the change of position of the object in the images were used to evaluate the stability of the sUAV. The rectilinear motion test evaluated the performance of the sUAVs as it follows a straight line path. Image processing algorithms were developed to evaluate both tests. Results showed that for the hovering test, both 
sUAV deviated \pm 1 meter while hovering, while the same deviation was observed in the straight line motion test. The sUAVs were also used to survey an experimental apple orchard and results showed that both sUAVs can be used for agricultural surveying.

Acknowledgments: This research was supported by the Idaho State Department of Agriculture (Idaho Specialty Crop Block Grant 2014) and Northwest Nazarene University.

Author Contributions: Duke M. Bulanon conceived the study, made the literature review, designed the experiment, processed the data, interpreted the results and wrote the paper. Esteban Cano, Ryan Horton and Chase Liljegren acquired the images, developed the image processing algorithm, and processed the data.

Conflicts of Interest: The authors declare no conflict of interest.

\section{Abbreviations}

The following abbreviations are used in this manuscript:

DIY: Do it yourself

GIS: Geographical Information System

GPS: Global Positioning System

NGB: Near-infrared, Green, Blue

NNU: Northwest Nazarene University

PVC:Polyvinyl Chloride

RGB: Red, Green, Blue

sUAV: Small Unmanned Aerial Vehicle

UAV: Unmanned Aerial Vehicle

VI: Vegetation Index

\section{References}

1. Robert, P.C. Precision agriculture: a challenge for crop nutrition management. Plant and Soil. 2002, 247, 143149

2. Lee, W.S.; Alchanatis, V.; Yang, C.; Hirafuji, M.; Moshou, D.; Li, C. Sensing technologies for precision specialty crop production. Comput. Electron. Agric. 2010, 74, 2-33

3. Koch, B.; Khosla, R. The Role of Precision Agriculture in Cropping Systems. J. Crop Prod. 2003, 9, 361-381

4. Seelan, S.K.; Laguette, S.; Casady, G.M.; Seielstad, G.A. Remote sensing applications for precision agriculture: A learning community approach. Remote Sens. of Environ. 2003, 88, 157-169

5. Sugiura, R.; Noguchi, N.; Ishii, K. Remote-sensing technology for vegetation monitoring using an unmanned helicopter. Biosyst. Eng. 2005, 90, 369-379.

6. Zhang, X.; Seelan, S.; Seielstad, G. Digital Northern Great Plains: A Web-Based System Delivering Near Real Time Remote Sensing Data for Precision Agriculture. Remote Sens. 2010, 2, 861-873

7. Albergel, C.; De Rosnay, P.; Gruhier, C.; Munoz-Sabater, J.; Hasenauer, S.; Isaken, L.; Kerr, Y., Wagner, W. Evaluation of remotely sensed and modelled soil moisture products using global ground-based in situ observations. Remote Sens. of Environ. 2012, 118, 215-226

8. Pajares, G. Overview and current status of remote sensing applications based on Unmanned Aerial Vehicles (UAVs). Photogramm. Eng. Remote Sen. 2015, 81, 281-329.

9. Thomasson, J.A.; Valasek, J. Small UAS in Agricultural Remote-Sensing Research at Texas A\&M. ASABE Resour. Magazine. 2016, 23(4), 19-21

10. Lan, Y.; Thomson, S.J.; Huang, Y.; Hoffmann, W.C.; Zhang, H. Current status and future directions of precision aerial application for site-specific crop management in the USA. Comput. Electron. Agric. 2010, 74, 34-38.

11. Garcia-Ruiz, F.; Sankaran, S.; Maja, J.M.; Lee, W.S.; Rasmussen, J.; Ehsani, R. Comparison of two aerial imaging platforms for identification of Huanglongbing-infected citrus trees. Comput. Electron. Agric. 2013, 91, 106-115.

12. Bulanon, D.M.; Lonai, J.; Skovgard, H.; Fallahi, E. Evaluation of Different Irrigation Methods for an Apple Orchard Using an Aerial Imaging System. ISPRS Int. J. Geo-Inf. 2016, 5(6), 79

13. Dulo, D.A. UNMANNED AIRCRAFT CLASSIFICATION - The Foundation for UAS Regulations in the National Airspace. The SciTech Lawyer. 2015, 11(4), 16-23 
14. Federal Aviation Administration. Available online: https://registermyuas.faa.gov (accessed on 29 June 2016).

15. 3DR. Available online: https://store.3dr.com/products/IRIS (accessed on 29 June 2016)

16. DJI. Available online: http://www.dji.com/product/phantom-2 (accessed on 29 June 2016)

17. Xiang, H; Tian, L. Development of a low-cost agricultural remote sensing system based on an autonomous unmanned aerial vehicle (UAV). Biosystems Engineering. 2011, 108, 174-190

18. Gonzalez, R.C., \& Woods, R.E. (2007). Digital Image Processing (3 ${ }^{\text {rd }}$ ed). New York City, New York: Pearson

(C) 2016 by the authors; licensee Preprints, Basel, Switzerland. This article is an open access article distributed under the terms and conditions of the Creative Commons by Attribution (CC-BY) license (http://creativecommons.org/licenses/by/4.0/). 